\title{
Topological multicritical point in the phase diagram of the toric code model and three-dimensional lattice gauge Higgs model
}

\author{
I. S. Tupitsyn, ${ }^{1,2}$ A. Kitaev, ${ }^{3}$ N. V. Prokof'ev, ${ }^{4}$ and P. C. E. Stamp ${ }^{1,2}$ \\ ${ }^{1}$ Pacific Institute of Theoretical Physics, Vancouver, British Columbia, Canada V6T 1Z1 \\ ${ }^{2}$ Department of Physics and Astronomy, University of British Columbia, Vancouver, British Columbia, Canada V6T 1Z1 \\ ${ }^{3}$ California Institute of Technology, Pasadena, California 91125, USA \\ ${ }^{4}$ Department of Physics, University of Massachusetts, Amherst, Massachusetts 01003, USA
}

(Received 14 June 2010; published 17 August 2010)

\begin{abstract}
We construct a mapping between the two-dimensional toric code model in external magnetic fields, $h_{z}$ and $h_{x}$, and the three-dimensional classical Ising system with plaquette interactions, which is equivalent to the three-dimensional $\mathbb{Z}_{2}$ gauge Higgs model with anisotropy between the imaginary time and spatial directions. The isotropic limit of the latter model was studied using Monte Carlo simulations on large (up to $60^{3}$ ) lattices in order to determine the stability of the topological phase against generic magnetic field perturbations and to resolve fine details of the phase diagram. We find that the topological phase is bounded by second-order transition lines, which merge into a first-order line at what appears to be a multicritical point arising from the competition between the Higgs and confinement transitions in the $Z_{2}$ gauge system. An effective field theory for this type of multicritical point (if one actually exists) is not known. Our results have potential applications to frustrated magnets, quantum computation, lattice gauge models in particle physics, and critical phenomena.
\end{abstract}

DOI: 10.1103/PhysRevB.82.085114

PACS number(s): 05.50.+q, 03.65.Vf, 05.30.Pr, 05.70.Jk

\section{INTRODUCTION}

Topological quantum phases with anyon excitations are well known in connection with the fractional quantum-Hall effect but they are also expected to exist in frustrated magnetic systems. It has long been proposed that a certain class of resonating-valence-bond ${ }^{1}$ phases carries $\mathbb{Z}_{2}$ charges and vortices, and has a fourfold degenerate ground state on a torus. ${ }^{2}$ Qualitative understanding of this phase can be obtained from the toric code model (TCM). ${ }^{3}$ The dimer model on the Kagome lattice is mapped onto the TCM exactly ${ }^{4}$ while some other models ${ }^{5,6}$ belong to the same universality class.

The TCM is defined in terms of spin- $1 / 2$ degrees of freedom located on bonds of the two-dimensional (2D) square lattice

$$
H_{T C}=-J_{x} \sum_{s} A_{s}-J_{z} \sum_{p} B_{p}
$$

where $A_{s}=\prod_{j \in s} \sigma_{j}^{x}$ and $B_{p}=\prod_{j \in p} \sigma_{j}^{z}$ are products of spin operators ( $\sigma_{j}^{\alpha}$ are the Pauli matrices) on the bonds incident to a site $s$ and on the boundary of a plaquette $p$, respectively. The ground state corresponds to eigenvalues $A_{s}=1, B_{p}=1$ for all $s$ and $p$. On a surface of genus $g$, it is $4^{g}$-fold degenerate. Elementary excitations are characterized by eigenvalues $A_{s}=-1\left(\mathrm{a} Z_{2}\right.$ charge on site $\left.s\right)$ and $B_{p}=-1$ (a $Z_{2}$ vortex on plaquette $p$ ); all excitations are gapped. Each type of quasiparticle is bosonic, but due to nontrivial mutual braiding, they must be jointly regarded as Abelian anyons.

Hamiltonian (1) has special properties related to its exact solvability: the two-point correlators vanish and the quasiparticle dispersion is flat. These features do not survive a small generic perturbation, while the topological character of the ground state and the anyonic statistics of quasiparticles remain robust. Yet, a sufficiently strong field can polarize the spins, driving a transition to the topologically trivial phase.
Trebst et al..$^{7}$ studied a magnetic field perturbation $-h_{z} \Sigma_{b} \sigma_{b}^{z}$ and solved the problem by reducing it to the $2 \mathrm{D}$ transversefield Ising model which was then mapped to an anisotropic three-dimensional (3D) classical Ising system. In this work, we consider a more general Hamiltonian

$$
H_{Q}=H_{T C}-h_{x} \sum_{b} \sigma_{b}^{x}-h_{z} \sum_{b} \sigma_{b}^{z},
$$

where $b$ runs over the lattice bonds and $H_{T C}$ is given by Eq. (1). Note that the fields $h_{x}$ and $h_{z}$ induce different types of phase transitions. The $h_{z}$ term creates virtual pairs of $\mathbb{Z}_{2}$ charges, which condense when the field strength exceeds a certain threshold. This phenomenon may be described as a Higgs transition, or vortex confinement. By duality, the $h_{x}$ field causes condensation of vortices and charge confinement. The competition of the two terms results in an interesting quantum phase diagram.

We approach the problem by mapping the $2 \mathrm{D}$ quantum problem in Eq. (2) onto a classical problem in one dimension higher, see Eq. (9). The resulting model contains four parameters and is classified as an anisotropic $Z_{2}$ gauge Higgs model. The universal properties of continuous transitions are likely to be independent of the anisotropy (between the imaginary time and spatial directions). We perform the actual numerics for the isotropic case, i.e., for the $M_{3,2}$ model as defined by Wegner, ${ }^{8}$ in order to reduce the number of free parameters and to speed up the computation.

Some properties of the phase diagram of the isotropic 3D Higgs model were predicted by Fradkin and Shenker. ${ }^{9}$ In particular, the topological phase is bounded by second-order lines described by charge condensation (for $h_{x} \ll h_{z} \sim J_{x}, J_{z}$ ) and vortex condensation (for $h_{z} \ll h_{x} \sim J_{x}, J_{z}$ ). While the two transitions are distinct as approached from the topological phase, there is no fundamental difference between the resulting condensates, which can be continuously transformed one 
to the other. For Hamiltonian (2), a connecting path is realized by increasing $h_{z}$ to polarize the spins in the $z$ direction, rotating the polarizing field in the $x z$ plane, and then decreasing the $h_{x}$ field. However, the two transition lines are not expected to join smoothly.

The first numerical study of the isotropic $\mathbb{Z}_{2}$ gauge Higgs model involving $10^{3}$ sites by Jongeward, Stack, and Jayaprakash ${ }^{10}$ showed that the second-order lines terminate in the vicinity of a finite first-order transition interval that partially separates the charge and vortex condensates. The conjecture was that the second-order lines might become first-order before merging. Subsequent simulations on lattices with up to $30^{3}$ sites by Genovese et al. ${ }^{11}$ revealed strong finite-size effects in this region of the phase diagram and rejected the conjecture. The actual topology of connections between the lines remained unanswered, leaving three possible scenarios: (1) a single point where all three lines end, (2) disconnected first-order line, and (3) termination of second-order transitions at the first-order line, see inset in Fig. 2.

In this work we attempted simulations of even larger systems (up to $60^{3}$ sites) in order to solve the puzzle. In agreement with Ref. 11, we rule out the original conjecture. ${ }^{10}$ Nevertheless, more accurate data and better resolution in the parameter space did not allow us to differentiate conclusively between the remaining scenarios. In the likely case of first scenario, we argue that the point where all three lines end is a novel type of multicritical point. Note that each of the second-order transitions is characterized by an Ising-type order parameter representing the magnitude of the corresponding condensate. The two orders must somehow coexist at the multicritical point though they are incompatible at the semiclassical level due to the nontrivial braiding between charges and vortices.

\section{QUANTUM-TO-CLASSICAL MAPPING}

Hamiltonian (2) is not gauge invariant but it can be mapped to a $Z_{2}$ gauge theory by introducing a dummy spin variable $\mu_{s}$ (matter field) at each site. In the enlarged Hilbert space, we only consider states $|\Psi\rangle$ such that $\mu_{s}^{x}|\Psi\rangle=|\Psi\rangle$. This constraint is a prototype of the gauge-theoretic condition stating that physical states are invariant under the operator $\mu_{s}^{x} A_{s}$, which flips the spins on site $s$ and all incident bonds. To turn one constraint into the other, we apply the transformation

$$
\begin{array}{cc}
\sigma_{u v}^{z} \rightarrow \mu_{u}^{z} \sigma_{u v}^{z} \mu_{v}^{z} & \sigma_{u v}^{x} \rightarrow \sigma_{u v}^{x} \\
\mu_{s}^{z} \rightarrow \mu_{s}^{z} & \mu_{s}^{x} \rightarrow \mu_{s}^{x} A_{s},
\end{array}
$$

where $\sigma_{u v}$ describes spins on the bond $b$ connecting sites $u$ and $v$. The transformed Hamiltonian has this form

$$
H=-J_{x} \sum_{s} \mu_{s}^{x}-J_{z} \sum_{p} B_{p}-h_{x} \sum_{b} \sigma_{b}^{x}-h_{z} \sum_{u v} \mu_{u}^{z} \sigma_{u v}^{z} \mu_{v}^{z}
$$

Note that in the first term, we have replaced $A_{s}$ by $\mu_{s}$ using the condition that $\mu_{s}^{x}|\Psi\rangle=A_{s}|\Psi\rangle$ for all physical states $|\Psi\rangle$.

We now map this $2 \mathrm{D}$ quantum Hamiltonian onto a $(2+1)$-dimensional classical model. The overall approach is

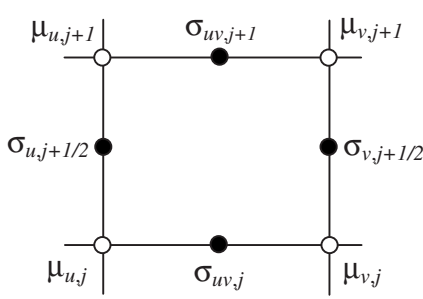

FIG. 1. Physical spins $\sigma_{u v, j}$ and auxiliary spins $\mu_{u, j}, \sigma_{u, j+1 / 2}$ on the $3 \mathrm{D}$ simple cubic lattice.

standard $^{12}$ and based on imaginary time evolution, with special care taken to preserve the gauge invariance. We divide the imaginary time interval $[0, \beta]$ into a large number of smaller intervals, $\Delta \tau=\beta / n$, and use the Trotter formula to express the quantum partition function $Z=\operatorname{Tr}[\exp (-\beta H) \mathcal{P}]$, where $\mathcal{P}$ is the projector onto the gauge-invariant subspace. Namely, we approximate the operator $\exp (-\beta H)$ by $\left[\exp \left(-\Delta \tau H_{x}\right) \exp \left(-\Delta \tau H_{z}\right)\right]^{n}$, where $H_{x}$ and $H_{z}$ are the terms in the quantum Hamiltonian that depend on $\sigma_{b}^{x}, \mu_{s}^{x}$ and $\sigma_{b}^{z}$, $\mu_{s}^{z}$, respectively. The operator $\exp \left(-\Delta \tau H_{x}\right) \exp \left(-\Delta \tau H_{z}\right)$ represents a transition between two adjacent time slices, say, $j$ and $j+1$, and all matrix elements may be expressed in the standard basis, where $\sigma_{b}^{z}$ and $\mu_{s}^{z}$ are diagonal. This allows us to interpret the trace as the partition function of a classical system defined in terms of Ising variables $\sigma_{b, j}, \mu_{s, j}= \pm 1$ residing on the horizontal bonds and the sites of a space-time cubic lattice. More specifically, the operator $\exp \left(-\Delta \tau H_{z}\right)$ determines the statistical weight of spin configurations on each time slice, whereas $\exp \left(-\Delta \tau H_{x}\right)$ connects adjacent slices. The effect of the transverse field on a single spin is given by the matrix element

$$
\left\langle\sigma_{b, j+1}^{z}\left|e^{h_{x} \Delta \tau \sigma_{b}^{x}}\right| \sigma_{b, j}^{z}\right\rangle=\left[\frac{1}{2} \sinh \left(2 \tilde{h}_{x}\right)\right]^{1 / 2} e^{\gamma_{x} \sigma_{b, j+1}^{z} \sigma_{b, j}^{z}},
$$

where $\tilde{h}_{x}=h_{x} \Delta \tau$ and $\gamma_{x}=-(1 / 2) \ln \tanh \left(\tilde{h}_{x}\right)$. A similar transformation applied to the $-J_{x} \mu_{s}^{x}$ term introduces a coupling constant $\delta_{x}=-(1 / 2) \ln \tanh \left(\tilde{J}_{x}\right)$ with $\widetilde{J}_{x}=J_{x} \Delta \tau$ between matter fields on the two time slices. Thus, the discrete imaginary time evolution corresponds to the following classical Hamiltonian:

$$
\begin{aligned}
H_{C}= & -\delta_{x} \sum_{u, j} \mu_{u, j} \mu_{u, j+1}-\tilde{J}_{z} \sum_{p, j} B_{p, j}-\gamma_{x} \sum_{u v, j} \sigma_{u v, j} \sigma_{u v, j+1} \\
& -\tilde{h}_{z} \sum_{u v} \mu_{u, j} \sigma_{u v, j} \mu_{v, j},
\end{aligned}
$$

where $\widetilde{J}_{z}=J_{z} \Delta \tau$ and $\tilde{h}_{z}=h_{z} \Delta \tau$.

Equation (5), however, lacks symmetry between space and time directions since vertical bonds [between sites $(u, j)$ and $(u, j+1)]$ do not carry any spins. In addition, we need to insert the gauge projector $\mathcal{P}$. To address both issues, we write the partition function as $\left[\exp \left(-\Delta \tau H_{x}\right) \mathcal{P} \exp \left(-\Delta \tau H_{z}\right)\right]^{n}$ (note that $\mathcal{P}$ commutes with both $H_{x}$ and $H_{z}$ ) and introduce summation over auxiliary spin variables $\sigma_{u, j+1 / 2}$ on vertical bonds (see Fig. 1), which represent the choice of a term in the expansion of $\mathcal{P}=\Pi_{u}\left[\frac{1}{2}\left(1+\mu_{u}^{x} A_{u}\right)\right]$. Up to the constant factor $(1 / 2)^{k}$, the replacement of $\exp \left(-\Delta \tau H_{x}\right)$ with $\exp \left(-\Delta \tau H_{x}\right) \mathcal{P}$ is 
equivalent to the following substitution in Eq. (5):

$$
\begin{gathered}
\mu_{u, j} \mu_{u, j+1} \rightarrow \mu_{u, j} \mu_{u, j+1} \sigma_{u, j+1 / 2}, \\
\sigma_{u v, j} \sigma_{u v, j+1} \rightarrow \sigma_{u v, j} \sigma_{u v, j+1} \sigma_{u, j+1 / 2} \sigma_{u v, j+1 / 2} .
\end{gathered}
$$

Thus we arrive at the following classical Hamiltonian:

$$
\begin{gathered}
H_{C}=-\sum_{u v} \lambda_{\text {bond }}^{\|, \perp} \mu_{u} \sigma_{u v} \mu_{v}-\sum_{\mathrm{p}} \lambda_{\mathrm{pl}}^{\|, \perp} \prod_{j \in \mathrm{p}} \sigma_{j}, \\
\lambda_{\text {bond }}^{\|}=-\frac{1}{2} \ln \tanh \widetilde{J}_{x} \quad-\text { vertical bonds, } \\
\lambda_{\text {bond }}^{\perp}=\tilde{h}_{z} \quad-\text { horizontal bonds, } \\
\lambda_{\mathrm{pl}}^{\|}=-\frac{1}{2} \ln \tanh \tilde{h}_{x} \quad-\text { vertical plaquettes, } \\
\lambda_{\mathrm{pl}}^{\perp}=\tilde{J}_{z} \quad-\text { horizontal plaquettes. }
\end{gathered}
$$

This model is an anisotropic generalization of the $\mathbb{Z}_{2}$ gauge Higgs model. ${ }^{9}$

As a final step, we eliminate the redundancy by fixing $\mu_{u, j}$. This only changes the classical partition function by a constant factor since Hamiltonian (7) can be written in terms of the gauge-invariant variables $S_{u v}=\mu_{u} \sigma_{u v} \mu_{v}$

$$
\tilde{H}_{C}=-\sum_{b} \lambda_{\text {bond }}^{\|, \perp} S_{b}-\sum_{\mathrm{p}} \lambda_{\mathrm{pl}}^{\|, \perp} \prod_{j \in \mathrm{p}} S_{j} .
$$

Taking all constant factors in the above transformations into account, we find the exact relation between (the discrete versions of) the quantum and classical partition functions

$$
Z=\left[\frac{1}{2} \sinh \left(2 \widetilde{J}_{x}\right)\right]^{k / 2}\left[\frac{1}{2} \sinh \left(2 \tilde{h}_{x}\right)\right]^{m / 2} \tilde{Z}_{C},
$$

where $k$ and $m$ are the numbers of vertical bonds and vertical plaquettes, respectively.

Model (9) can be considered as an anisotropic generalization of the $Z_{2}$ gauge Higgs system ${ }^{9}$ and contains four free parameters. Although the derivation was done in the limit of small $\Delta \tau$, we believe that the qualitative phase diagram and the nature of critical points are universal. In what follows we consider the isotropic version of the model

$$
\tilde{H}_{C}=-\lambda_{\text {bond }} \sum_{b} S_{b}-\lambda_{\mathrm{pl}} \sum_{\mathrm{p}} \prod_{j \in \mathrm{p}} S_{j},
$$

where $\lambda_{\text {bond }}=\tilde{h}_{z}=-\frac{1}{2} \ln \tanh \tilde{J}_{x}$ and $\lambda_{\mathrm{pl}}=\tilde{J}_{z}=-\frac{1}{2} \ln \tanh \tilde{h}_{x}$.

\section{PHASE DIAGRAM IN THE ISOTROPIC CASE}

At $\lambda_{\text {bond }}=0$ all configurations, including ground states, have the same degeneracy factor $2^{2 N}$. The actual physical variables in this limit are plaquette numbers $N_{p}=\prod_{j \in \mathrm{p}} S_{j}$, and the model itself is dual to the 3D classical Ising model [Eq. (11) is also known as the $3 \mathrm{D}$ Ising gauge theory $\left.{ }^{8}\right]$. Using high-accuracy results of Ref. 13 for the critical point and the

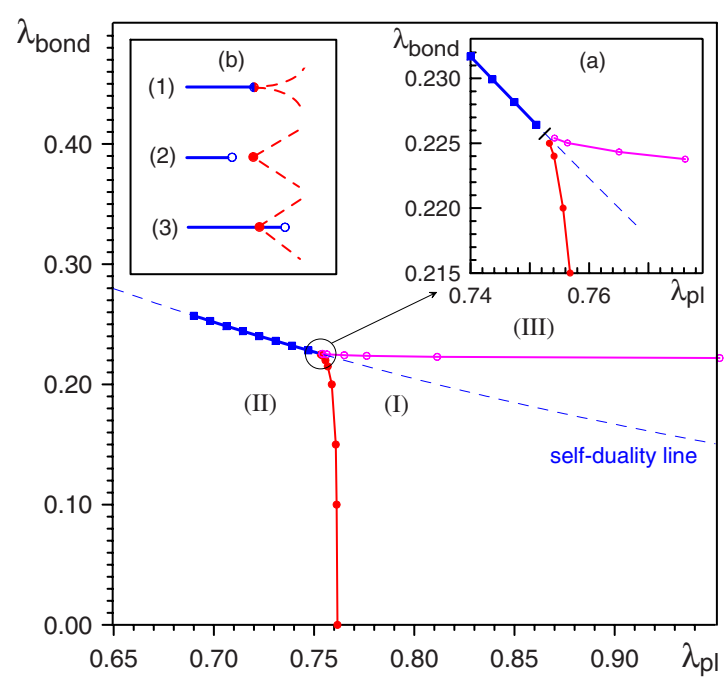

FIG. 2. (Color online) The phase diagram of Hamiltonian (11). Circles correspond to second-order transitions (open and filled symbols are related by the duality transformation). Filled squares mark a first-order transition on the self-duality line. The phases are: (I) topological phase; (II)—disordered phase; and (III)—magnetically ordered phase. In inset (a) we show the region where all phases meet each other. In inset (b) we show three alternative ways of connecting the lines; bold and dashed lines correspond to the firstand second-order transitions, respectively.

duality relation $\lambda_{\mathrm{pl}}=-1 / 2 \ln \tanh (J / T)$, where $J$ is the Ising exchange coupling, we obtain $\lambda_{\mathrm{pl}}^{(c)}=0.7614125$.

At arbitrary values of $\lambda_{\text {bond }}$ and $\lambda_{\mathrm{pl}}$ the model is self-dual, ${ }^{14}$ i.e., it maps to itself under the coupling constant transformation $\lambda_{\text {bond,pl }} \rightarrow-1 / 2 \ln \tanh \left(\lambda_{\text {pl,bond }}\right)$. This means that the phase diagram has a symmetry across the self-duality line defined by $\lambda_{\text {bond }}=-1 / 2 \ln \tanh \left(\lambda_{\mathrm{pl}}\right)$. Under the duality mapping $\left(\lambda_{\text {bond }}=0, \lambda_{\mathrm{pl}}=0.7614125\right)$ $\rightarrow\left(\lambda_{\text {bond }}=0.221655, \lambda_{\mathrm{pl}}=\infty\right)$, which gives us two Ising-type critical points on the phase diagram.

To calculate the rest of the phase diagram we performed Monte Carlo (MC) simulations using standard single-spin flip updates, supplemented by rare (once per $N^{2}$ updates) flips of all spins belonging to bonds cut by planes oriented along any one of the crystal axes or along any of the diagonals to these axes. There are $9 N$ possible planes satisfying this condition, and we select any of them at random. The plaquette energy [second term in Eq. (11)] is conserved by this update. To determine the second-order critical lines, we employed the standard finite-size scaling analysis of the specific heat $C_{v}$, for linear system sizes $N=24,36,48$, and 60 (i.e., for $3 N^{3}$ spins). First-order critical points were identified and located using energy distributions. These distributions are bimodal (have two maxima) at the first-order transition and single-modal otherwise. We anneal our samples before collecting statistics for up to $10^{6} \mathrm{MC}$ sweeps (one sweep having $3 N^{3}$ elementary updates). The data were accumulated for $\sim 4 \times 10^{8} \mathrm{MC}$ sweeps.

The resulting phase diagram is presented in Fig. 2. The first-order transition coinciding with the self-duality line was observed for $0.2575(5)>\lambda_{\text {bond }}>0.22635(5)$. Outside of this interval we did not detect any bimodal structure in the energy 


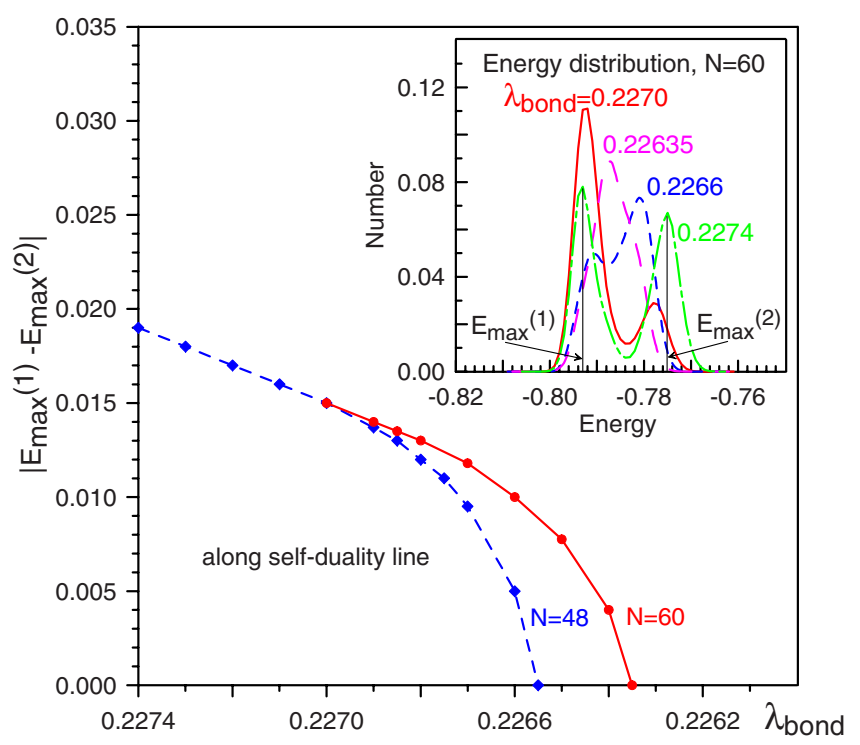

FIG. 3. (Color online) Energy splitting between two maxima in the bimodal energy distributions along the self-duality line for $N=48$ and $N=60$ as a function of $\lambda_{\text {bond. }}$. The inset shows examples of energy distributions at various values of $\lambda_{\text {bond }}$.

distribution for system sizes up to $N=60$. The inset of Fig. 3 shows the evolution of the energy distribution function along the self-dual line. Even when the bimodal structure is observed, it is extremely weak, developing only for large $N$. Under these conditions, the distribution can be sampled across the minimum without the flat-histogram or similar reweighing techniques.

As noted above, these results disagree with conclusions of Ref. 10 that the first-order line splits into two first-order transitions. The inset (a) in Fig. 2 shows a closeup of the controversial region. Though we were able to resolve transition points with accuracy of at least three digits, we observed no evidence for splitting of the self-dual first-order line into two first-order transitions. We also find no evidence for tricritical points on the Ising-type lines as long as we can resolve two separate transitions. There remains a tiny parameter range between the apparent disappearance of the bimodal distribution on the self-dual line (this disappearance is likely due to limited system sizes) and two resolved second-order transitions.

To probe the behavior in this tiny parameter range one has to use a different strategy. We scanned the energy distributions (for $N=48$ ) at 30 points along the line perpendicular to the self-duality line right in the questionable region [short solid line in the inset (a)]. If the first-order line were to split above the scan, the third maximum would have to emerge in the energy distribution in between the two maxima we observe on the self-dual line-implying that the energy maxima on the self-dual line could not merge smoothly, and, right below the split, three maxima would have to be seen in the energy distribution. However all distributions along the scan were found to have only one peak. It is also clear from the main plot in Fig. 3 that on the self-dual line the energy maxima approach each other and merge continuously as $\lambda_{\text {bond }}$ decreases. The curves presented in Fig. 3 follow a power law near the vanishing point, with the corresponding critical exponent $\sim 0.55$.

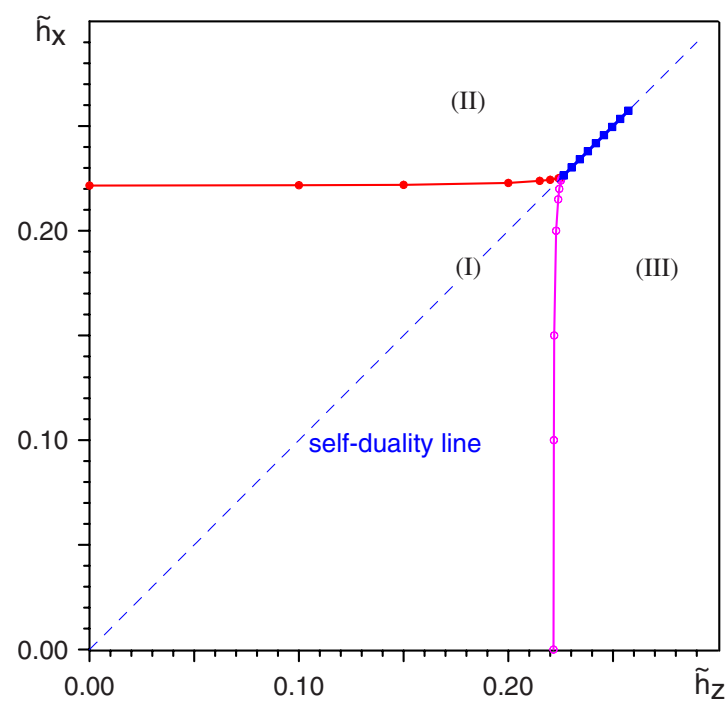

FIG. 4. (Color online) The phase diagram of Fig. 2 in terms of the renormalized external fields, $\tilde{h}_{x}=h_{x} \Delta \tau$ and $\tilde{h}_{z}=h_{z} \Delta \tau$ of Hamiltonian (2). The phases (i), (II), and (II) are the same as in Fig. 2.

We thus conclude, in agreement with Ref. 11, that the first-order line does not split into two first-order transitions. Instead, there are three other possibilities. Either all three lines merge at one point [case (1) in the inset (b), Fig. 2]; or the first-order line ends before or after the point where two second-order lines touch the self-dual line [cases (2) and (3) in the inset (b), Fig. 2]. Unfortunately, our data cannot distinguish between the alternatives because the second-order lines seem to touch at extremely small (possibly zero) angle. Theoretically, the last scenario is the least demanding since it fits the existing theory of phase transitions. Speaking of the second case, our data indicate that the second-order lines do not join smoothly but rather form a cusp at the self-duality line. We are not aware of any effective field theory that would explain this or the first (the most likely) scenario.

\section{PHASES}

Using the correspondence equations

$$
\begin{gathered}
\tilde{h}_{z}=-\frac{1}{2} \ln \tanh \left(J_{x} \Delta \tau\right)=\lambda_{\text {bond }}, \\
\widetilde{J}_{z}=-\frac{1}{2} \ln \tanh \left(h_{x} \Delta \tau\right)=\lambda_{\mathrm{pl}}
\end{gathered}
$$

we can reformulate the phase diagram in Fig. 2 in terms of renormalized parameters $\widetilde{J}_{x}=J_{x} \Delta \tau, \widetilde{J}_{z}, \tilde{h}_{x}=h_{x} \Delta \tau$, and $\tilde{h}_{z}$ of the TCM. The resulting phase diagram in the $\left(\tilde{h}_{z}, \tilde{h}_{x}\right)$ plane is presented in Fig. 4. Phase (I) corresponds to the topological phase of model (2) [the "free charge" phase of the isotropic $\mathbb{Z}_{2}$ Higgs model (HM) (Ref. 9)]. In this phase, the system tends to have all $B_{p}=1$ and $A_{s}=1$ and a realization of such a state is obviously not unique. The plaquettes with $B_{p}=-1$ (magnetic vortices) and vertices with $A_{s}=-1$ (electric charges) appear mainly in the vicinity of the critical lines 
between the phases. The state (III) may be called "magnetically ordered" in the limit $\widetilde{h}_{x} \rightarrow 0$ when spins are mostly polarized in the $z$ direction. Since $\left\langle\sigma^{z}\right\rangle$ has nonzero value everywhere in the phase diagram, the true order parameter may be written as $\left\langle\mu^{z}\right\rangle$ using the gauge-symmetrized Hamiltonian (4). A nonzero value of this parameter results in the confinement of magnetic vortices (no free vortices) and the condensation of electric charges. In the HM this is the "Higgs" phase. The state (II) in the limit $\widetilde{h}_{z} \rightarrow 0$ is characterized by an order parameter dual $\left\langle\sigma^{x}\right\rangle$, which can be defined by rewriting the Hamiltonian in different variables. Its nonzero value results in nonconservation of the total magnetic "charge" and condensation of magnetic vortices while electric charges are confined (no free charges). This state corresponds to the "confinement" phase of the HM. While there is no fundamental distinction between (II) and (III) when both external fields are nonzero, there is a possibility of having a firstorder transition within the phase. In our case, the first-order transition is accompanied by an abrupt change in the number of vortices and charges, similar to the density discontinuity in the liquid-gas transition. The self-duality reflects the symmetry between charges and vortices.

\section{SUMMARY}

We examined the stability of the two-dimensional toric code model (a prototype system for topological quantum computation) against generic magnetic field perturbations. We mapped the TCM onto a 3D classical Ising-type model with four parameters. In the space-time symmetric case, we arrive at the $\mathbb{Z}_{2}$ gauge Higgs model on a simple cubic lattice and compute its phase diagram by employing large scale Monte Carlo simulations. The topological phase of the TCM (the free charge phase of the $\mathbb{Z}_{2}$ gauge Higgs model) remains stable in a wide range of field parameters and breaks down via two Ising-type transitions whose critical lines meet at (or in close vicinity of) the tip of a first-order line within the nontopological phase. An effective field theory describing the joining of these three lines remains an interesting open problem.

Recently, the effect of magnetic fields on TCM was studied perturbatively and by exact diagonalization for paralle ${ }^{15}$ and perpendicular ${ }^{16}$ fields by Vidal et al. The phase diagram, obtained in these works is qualitatively the same as ours including the apparent multicritical point. Phase diagrams of related models with both electric and magnetic confinements were studied in the past using various Monte Carlo techniques on smaller 3D lattices by Sedgevick et al. ${ }^{17}$ and Park and Sachdev. ${ }^{18}$ Despite an additional global $U(1)$ symmetry present in their systems, the topology of phase diagrams is similar to that obtained here.

\section{ACKNOWLEDGMENTS}

We thank E. Fradkin, B. Svistunov, S. Trebst, M. Troyer, I. Affleck, K. Shtengel, and S. Sachdev for discussions. We are also indebted to M. Berciu and J. Heyl whose research clusters were used to perform our MC simulations. N.P. acknowledges support from the National Science Foundation under Grant No. PHY-0653183, a grant from the Army Research Office with funding from the DARPA OLE program, and Aspen Center for physics.
${ }^{1}$ P. W. Anderson, Science 235, 1196 (1987).

${ }^{2}$ N. Read and B. Chakraborty, Phys. Rev. B 40, 7133 (1989).

${ }^{3}$ A. Yu. Kitaev, Ann. Phys. (N.Y.) 303, 2 (2003).

${ }^{4}$ G. Misguich, D. Serban, and V. Pasquier, Phys. Rev. Lett. 89, 137202 (2002).

${ }^{5}$ N. Read and S. Sachdev, Phys. Rev. Lett. 66, 1773 (1991).

${ }^{6}$ R. Moessner and S. L. Sondhi, Phys. Rev. Lett. 86, 1881 (2001).

${ }^{7}$ S. Trebst, P. Werner, M. Troyer, K. Shtengel, and Ch. Nayak, Phys. Rev. Lett. 98, 070602 (2007).

${ }^{8}$ F. J. Wegner, J. Math. Phys. 12, 2259 (1971).

${ }^{9}$ E. Fradkin and S. H. Shenker, Phys. Rev. D 19, 3682 (1979).

${ }^{10}$ G. A. Jongeward, J. D. Stack, and C. Jayaprakash, Phys. Rev. D 21, 3360 (1980).

${ }^{11}$ L. Genovese, F. Gliozzi, A. Rago, and C. Torrero, Nucl. Phys. B,
Proc. Suppl. 119, 894 (2003).

${ }^{12}$ M. Suzuki, Prog. Theor. Phys. 56, 1454 (1976).

${ }^{13}$ R. Gupta and P. Tamayo, US-Japan Bilateral Seminar-Maui, August 28-31, 1996.

${ }^{14}$ R. Balian, J. M. Drouffe, and C. Itzykson, Phys. Rev. D 11, 2098 (1975).

${ }^{15}$ J. Vidal, S. Dusuel, and K. P. Schmidt, Phys. Rev. B 79, 033109 (2009).

${ }^{16}$ J. Vidal, R. Thomale, K. P. Schmidt, and S. Dusuel, Phys. Rev. B 80, 081104 (2009).

${ }^{17}$ R. D. Sedgewick, D. J. Scalapino, and R. L. Sugar, Phys. Rev. B 65, 054508 (2002).

${ }^{18}$ K. Park and S. Sachdev, Phys. Rev. B 65, 220405 (2002). 